\title{
Efficient Intertemporal Allocations with Recursive Utility*
}

\author{
Bernard Dumas, ${ }^{\dagger}$ Raman Uppal ${ }^{\ddagger}$ and Tan Wang ${ }^{\S}$
}

September 1997

*We express our thanks to Darrell Duffie, Pierre-Yves Geoffard, Chenghu Ma and Philippe Weil for their help, to Larry Epstein for detailed comments, and to Stanley Zin for his encouragement. Wang acknowledges the support of the Social Sciences and Humanities Research Council of Canada.

${ }^{\dagger}$ HEC School of Management, 78351 Jouy-en-Josas Cedex, France; London Business School, NBER and CEPR; Email: dumas@gwsmtp.hec.fr.

${ }^{\ddagger}$ Faculty of Commerce and Business Administration, University of British Columbia and Sloan School of Management, MIT, 50 Memorial Drive E52-410, Cambridge, MA 02142, USA; Email: uppal@mit.edu.

$\S$ Faculty of Commerce and Business Administration, University of British Columbia, 2053 Main Mall, Vancouver, B.C. V6T 1Z2, Canada; Email: tan.wang@commerce.ubc.ca. 


\begin{abstract}
In this article, our objective is to determine efficient allocations in economies with multiple agents having recursive utility functions. Our main result is to show that in a multiagent economy, the problem of determining efficient allocations can be characterized in terms of a single value function (that of a social planner), rather than multiple value functions (one for each investor), as has been proposed thus far. We then show how this value function can be identified using the familiar technique of stochastic dynamic programming. We achieve these goals by first extending to a stochastic environment Geoffard's (1996) concept of variational utility and his result that variational utility is equivalent to recursive utility, and then using these results to characterize allocations in a multiagent setting.
\end{abstract}

JEL classification: D81, D61, D91, C61.

Key words: Variational utility, stochastic control, dynamic programming, Bellman equation 
Following Lucas and Stokey (1984), Epstein (1987) has characterized efficient (welfare-maximizing) allocations with recursive utility under certainty. Duffie, Geoffard and Skiadas (1994) have a similar result under uncertainty. Both formulations involve a multiplicity of unknown value functions, namely one for each consumer investor. In this article, we endeavor to reformulate these problems in such a way that they will involve only one value function, namely the maximized social welfare. This is done using the concept of "felicity function" introduced by Geoffard (1996). Furthermore, we show how dynamic programming can be used to implement that optimization.

The main contribution of our work is methodological: the results of this paper will allow economists to perform welfare analysis for economies with agents having recursive utility in the traditional way, that is, by optimizing aggregate social welfare. In many papers, welfare optima are calculated, not for themselves but as a short-cut in the calculation of a purely competitive market equilibrium. This approach to the general equilibrium of a competitive market was pioneered by Negishi (1960) in the finite-dimensional case and significantly extended by Magill (1981) and MasColell (1986) among others to the infinite-dimensional case. In view of this approach, our paper opens the path for a convenient method of computing equilibria in economies with multiple agents having recursive utility functions.

Recursive utility functions have one well-known advantage, viz. they allow a clean analysis of the comparative statics of risk. For instance, one can obtain simple answers to questions such as do savings increase or decrease when the level of risk of investment opportunities increases? In common parlance, recursive utility permits the disentangling of the two psychologically separate concepts of risk aversion (desire to stabilize consumption across states of nature) and elasticity of intertemporal substitution (desire to smooth consumption over time), which for the traditional time-additive utility functions are constrained to be equal to (the inverse of) each other. ${ }^{1}$ Even if it were true that, in the real world, each person's risk aversion were always exactly equal to the inverse of his/her elasticity of intertemporal substitution, it is still important to distinguish between the two concepts, in order to determine the size and direction of the effects of a change in the risks that investors face.

In Section 1, we provide some background to two methods adopted to extend time-additive expected utility: recursive utility and variational utility, the latter introduced by Geoffard (1996)

\footnotetext{
${ }^{1}$ Strictly speaking, risk aversion and elasticity of intertemporal substitution are not the inverse of each other in the general time-additive case. However, they are always related: the elasticity of intertemporal substitution between two points in time being equal to the inverse of a complex average of the two successive risk aversions. In the doubly isoelastic case (isoelasticity with respect to timeless consumption and isoelasticity with respect to time), they are indeed the inverse of each other. We use the word "inverse" as a figure of speech.
} 
for the case of certainty. In Section 2, we extend variational utility to the stochastic case and show that it allows an alternative formulation of recursive utility. In Section 3, we achieve our main goal, which is a simplified formulation, in continuous time and in a stochastic environment, of the Pareto optimality problem with recursive utility; the discrete time case is treated in an appendix. Section 4 contains an example that illustrates how the method proposed in this paper can be applied. Section 5 concludes.

\section{Background of Variational Utility}

Recursive utility was first axiomatized by Koopmans (1960) for the certainty case in discrete time. Epstein (1987) shows in continuous time, on the basis of five axioms concerning a person's utility of intertemporal consumption, $U\left({ }_{t} c\right)$, where ${ }_{t} c$ denotes the consumption stream starting at time $t$, that there exists an aggregator $f$, such that:

$$
\frac{d U\left({ }_{t} c\right)}{d t}=-f\left(c_{t}, U\left({ }_{t} c\right)\right)
$$

Epstein and Zin (1989) and Weil (1990) extend recursive utility to the uncertainty case in discrete time. Based on the work by Kreps and Porteus (1978), Epstein and Zin propose the recursive utility:

$$
V_{t}=W\left(c_{t}, \mu\left(V_{t+1}\right)\right)
$$

where $W$ is an aggregator and $\mu$ is a certainty equivalent operator. Duffie and Epstein (1992) extend stochastic recursive utility to continuous time, where it is defined as the solution to the equation

$$
V_{t}=E_{t}\left\{\int_{t}^{\infty} f\left(c_{s}, V_{s}\right) d s\right\}
$$

with the aggregator being $f$.

The above development of recursive utility followed the route of relaxing the intertemporally additive structure of expected utility. However, assuming certainty, Geoffard (1996) shows that there is an alternative equivalent approach that maintains the additive structure of expected utility. Following Geoffard's approach, we propose that in a stochastic environment the utility process $\left\{V_{t}\right\}$ be defined as the solution of

$$
V_{t}=F\left(c_{t}, \nu_{t}\right)+e^{-\nu_{t}} E_{t}\left[V_{t+1}\right],
$$


for some appropriate $\left\{\nu_{t}\right\}$. The function $F: R_{+} \times[-a, a] \rightarrow R$, strictly convex in its second argument, is called the "felicity function" by Geoffard (1996). The process $\left\{\nu_{t}\right\}$ is to be interpreted as the discount rate process. Utility processes satisfying (1) have the obvious intuitive appeal that the utility derived from a consumption process is equal to the sum of the discounted felicities of current and future consumptions

$$
V_{t}=E_{t}\left\{\sum_{s=t}^{\infty} e^{\sum_{j=t}^{s}-\nu_{j}} F\left(c_{s}, \nu_{s}\right)\right\} .
$$

Two familiar examples of such utility processes are the intertemporally additive expected utility and its extension by Uzawa:

$$
V_{t}=u\left(c_{t}\right)+e^{-\nu\left(c_{t}\right)} E_{t}\left[V_{t+1}\right]
$$

In the case of standard intertemporally-additive expected utility, the discount rate is typically assumed to be constant, while for Uzawa utility, $\nu_{t}=\nu\left(c_{t}\right)$. More generally, the discount rates in (2) can be allowed to depend on the entire consumption process.

In order for (2) to properly define a utility process one needs to specify the discount rate process, which can be done using discount rate functions. A discount rate function is a mapping $\nu$ from the space of all consumption processes to the space of all discount rate processes. It specifies, for any consumption process $\left\{c_{t}\right\}$, a discount rate process $\nu_{t}=\nu(c ; t)$. Subject to technical conditions, a discount rate function and equation (2) together define a utility process.

In this paper, we will focus on a particular class of discount rate functions that, together with equation (2), define the class of variational utility. Given the structure of (1), it seems natural to assume that the discount rate at time $t$ depends only on the current consumption level and the expected value of future utility, with the latter serving as a sufficient statistic for the utility derived from all future consumption. That is, there is a function $\nu: R_{+}^{2} \rightarrow R_{+}$such that

$$
\nu_{t}=\nu\left(c_{t}, E_{t}\left[V_{t+1}\right]\right)
$$

Specifically, we will focus on the class of discount rate functions implicitly defined by

$$
\nu_{t}=\arg \min _{\nu}\left[F\left(c_{t}, \nu\right)+(1-\nu) V_{t}\right]
$$

Discount rates generated by (3) are said to satisfy the Minimum Principle. ${ }^{2}$ To illustrate the intuition underlying the Minimum Principle, note that if $y_{1}$ and $y_{2}$ are cashflows in the current and

\footnotetext{
${ }^{2}$ The Minimum Principle stated in Geoffard (1996) is in the form of equation (9) below. It turns out that this is equivalent to (7) in the context of our paper.
} 
next period, and if the discount rate $r$ is given, then the present value of the cashflows is $y_{1}+\frac{1}{1+r} y_{2}$, which, when $r$ is small, is approximately

$$
y_{1}+(1-r) y_{2}
$$

Conversely, given the cash flows and their present value, $h\left(y_{1}, y_{2}\right) \equiv y_{1}+(1-r) y_{2}$, one can back out the discount rate as $r=1-h_{y_{2}}\left(y_{1}, y_{2}\right)$, where $h_{y_{2}}\left(y_{1}, y_{2}\right)$ denotes the derivative with respect to $y_{2}$. Thus, the present value function, $h\left(y_{1}, y_{2}\right)$, implicitly defines the discount rate.

By analogy, if $u$ is the second period utility and $W(c, u)$ is the "present value" of (the utility of) $c$ and $u$, then one can define the discount rate for utility as ${ }^{3}$

$$
\nu \equiv 1-W_{u}(c, u)
$$

As in (4), one can then express the present value of the utility flow as

$$
W(c, u)=F+(1-\nu) u
$$

where $F$ represents the contribution of $c$ to current-period utility and $\nu$ is a discount rate that satisfies (5). Equivalently, equations (5) and (6) can be expressed as:4

$$
F(c, \nu)=\max _{u}\{W(c, u)-(1-\nu) u\}
$$

Then by duality theory,

$$
W(c, u)=\min _{\nu}\{F(c, \nu)+(1-\nu) u\}
$$

which is the Minimum Principle in discrete time.

The continuous-time analog of the above discrete-time maximization problem is ${ }^{5}$

$$
\max _{u}\{f(c, u)+\nu u\}
$$

\footnotetext{
${ }^{3}$ Note, however, that since $W(c, u)$ is not necessarily linear in $(c, u)$, the discount rate may depend on $(c, u)$.

${ }^{4}$ Equation (5) is simply the first-order condition of the maximization problem.

${ }^{5}$ To see this, write the discrete-time maximization problem as

$$
\max _{u}\left\{W\left(c_{t-\Delta}, V_{t}\right)-(1-\nu \Delta) V_{t}\right\}=\max _{u}\left\{W\left(c_{t-\Delta}, V_{t}\right)-V_{t}+\nu \Delta V_{t}\right\} .
$$
}

By definition (see Epstein, 1987), then:

$$
f\left(c_{t}, V_{t}\right)=-\lim _{\Delta} \frac{W\left(c_{t-\Delta}, V_{t}\right)-V_{t}}{-\Delta} .
$$


and the corresponding Minimum Principle in continuous time is

$$
\min _{\nu}\{F(c, \nu)-\nu u\}
$$

Equation (7), coupled with the continuous-time version of (2),

$$
V_{t}=E_{t}\left\{\int_{t}^{T} e^{\int_{t}^{s}-\nu_{u} d u} F\left(c_{s}, \nu_{s}\right) d s\right\} \text {, }
$$

will form the basis of stochastic variational utility that we introduce below.

For readers familiar with the axiomatic approach to introducing utility functions, using the Minimum Principle to define discount rates may not seem entirely natural. The justification for using this approach then is the class of utility functions that it generates and the potential usefulness of this class. As Geoffard shows in the certainty case and as we will show later in the uncertainty case, variational utility identifies a class of utility functions that is familiar in the literature, namely, recursive utility. ${ }^{6}$ Thus, (7) and (8) provide an alternative method of characterizing recursive utility. Showing the usefulness of this alternative characterization is one objective of this paper.

\section{Stochastic Variational Utility}

In this section, we first extend to a stochastic environment the concept of variational utility developed by Geoffard (1996) under certainty. Then, we establish the relation between recursive and stochastic variational utility. We conclude this section by showing how, in a Markov setting, one can use dynamic programming to solve the optimization problem of a single agent with stochastic variational utility. The case of multiple agents is treated in Section 3.

\subsection{Definition of Stochastic Variational Utility}

Given the background and motivation for variational utility in Section 1, we now introduce the notion of stochastic variational utility. Let $\left(\Omega, \mathcal{F},\left\{\mathcal{F}_{t}\right\}, P\right)$ be a probability space with the filtration $\left\{\mathcal{F}_{t}: t \in[0, T]\right\}$ satisfying the usual conditions (increasing, right-continuous, augmented, and $\mathcal{F}_{0}$ being trivial). Let $D$ denote the space of processes $c: \Omega \times[0, T]$ that are right continuous and

\footnotetext{
${ }^{6}$ Strictly speaking, the variational utility introduced by Geoffard (1996) under certainty is more general than recursive utility. However, since we are interested in the connection between variational utility and recursive utility, and in demonstrating the potential usefulness of variational utility in solving some standard problems in economics, we will not pursue the most general formalization of the notion of variational utility in a stochastic setting.
} 
measurable with respect to the $\sigma$-algebra on $\Omega \times[0, T]$ generated by left-continuous and $\left\{\mathcal{F}_{t}\right\}$ adapted processes, and such that

$$
\|c\|=\left[E\left(\int_{0}^{T} c_{t}^{2} d t\right)\right]^{1 / 2}<\infty
$$

The positive cone of $D$ is denoted by $D_{+}$. The space of discount rate processes is defined as

$$
\Pi=\left\{\nu \in D: \nu_{t}(\omega) \in[-a, a], \forall(\omega, t) \in \Omega \times[0, T]\right\},
$$

where $a$ is a constant and serves as an upper bound on the absolute value of discount rates. Our discussion below will illustrate that this boundedness assumption is without loss of generality.

We consider a "felicity function," $F: D_{+} \times \Pi$, which in addition to being strictly convex in its second argument and continuously differentiable, has the following properties:

- $F$ is Lipschitz in the discount rate: there is a constant $k$ such that for all $c \in R_{+}$and all $v$ and $w \in[-a, a],|F(c, v)-F(c, w)| \leq k|v-w|$.

- F satisfies a linear growth condition in consumption: there are constants $k_{1}$ and $k_{2}$ such that for all $c \in R_{+}|F(c, 0)| \leq k_{1}+k_{2}|c|$.

As a convention, assumptions and properties apply to $F$ only in its effective domain: $\{(c, \nu)$ : $F(c, \nu)<\infty\}$.

Now we are ready to define Stochastic Variational Utility (SVU).

Definition 1 Let $c \in D_{+}$be a consumption process. The process $\left\{V_{t}\right\}$ is defined as the stochastic variational utility (process) of $c$ if there exists a discount rate process $\left\{\nu_{t}\right\} \in \Pi$ such that $\left(\left\{V_{t}\right\},\left\{\nu_{t}\right\}\right)$ solves equations (7) and (8).

Although (7) and (8) are the intuitive equations that one would use to define variational utility, it will prove more convenient to write variational utility in an alternative equivalent form. We do this in the following lemma. 
Lemma 1 Let $c \in D_{+}$be a consumption process. Then $\left(\left\{V_{t}\right\},\left\{\nu_{t}\right\}\right)$ is a solution to equations (7) and (8) if and only if it solves the following problem: for all $t$,

$$
\lambda_{t} V_{t}(c)=\inf _{\nu \in \Pi} E_{t}\left\{\int_{t}^{T} \lambda_{s} F\left(c_{s}, \nu_{s}\right) d s\right\} \quad \text { a.s. }
$$

subject to

$$
d \lambda_{s}=-\nu_{s} \lambda_{s} d s, \quad \lambda_{0}=1
$$

where $\left\{\lambda_{t}\right\}$ is called the discount factor process. Since the value function of a minimization problem is unique, the variational utility of $c$, if it exists, is well-defined.

Proof: Let $c \in D_{+}$. Suppose that $\left(\left\{V_{s}(c)\right\},\left\{\nu_{s}^{*}\right\}\right)$ is a solution to the minimization problem (9). Then (8) is clearly satisfied. To show (7), let $\left\{\nu_{s}\right\}$ be a discount rate process such that for $s \geq t^{\prime}$, $\nu_{s}=\nu_{s}^{*}$, but for $s<t^{\prime}$ it is arbitrary except for the right continuity, measurability and integrability conditions. Let $\lambda$ and $\lambda^{*}$ be the discount factor processes associated with $\nu$ and $\nu^{*}$, respectively. Then for any $t<t^{\prime}$,

$$
\lambda_{t} V_{t}(c) \leq E_{t}\left\{\int_{t}^{t^{\prime}} \lambda_{s} F\left(c_{s}, \nu_{s}\right) d s+\lambda_{t^{\prime}} V_{t^{\prime}}(c)\right\}
$$

¿From $(9),\left\{\lambda_{t}^{*} V_{t}(c)\right\}$ is a semimartingale of class $D$. Hence,

$$
\lambda_{t}^{*} V_{t}(c)=V_{0}(c)-\int_{0}^{t} \lambda_{s}^{*} F\left(c_{s}, \nu_{s}^{*}\right) d s+M_{t}
$$

where $M_{t}$ is a square-integrable martingale. By Itô's formula (Protter, 1990),

$$
\lambda_{t^{\prime}} V_{t^{\prime}}(c)=\lambda_{t} V_{t}(c)-\int_{t}^{t^{\prime}}\left(\nu_{s}-\nu_{s}^{*}\right) \lambda_{s} V_{s}(c) d s-\int_{t}^{t^{\prime}} \lambda_{s} F\left(c_{s}, \nu_{s}^{*}\right) d s+\int_{t}^{t^{\prime}} \frac{\lambda_{s}}{\lambda_{s}^{*}} d M_{s}
$$

Thus,

$$
0 \leq E_{t}\left\{\int_{t}^{t^{\prime}} \lambda_{s} F\left(c_{s}, \nu_{s}\right) d s-\int_{t}^{t^{\prime}}\left(\nu_{s}-\nu_{s}^{*}\right) \lambda_{s} V_{s}(c) d s-\int_{t}^{t^{\prime}} \lambda_{s} F\left(c_{s}, \nu_{s}^{*}\right) d s\right\} .
$$

Taking limits and noting the right continuity of the processes, yields

$$
0 \leq\left[F\left(c_{t}, \nu_{t}\right)-\nu_{t} V_{t}(c)\right]-\left[F\left(c_{t}, \nu_{t}^{*}\right)-\nu_{t}^{*} V_{t}(c)\right]
$$

which is $(7)$ since $\nu_{t}$ is arbitrary. 
For the converse, suppose $\left(\left\{V_{s}(c)\right\},\left\{\nu_{s}^{*}\right\}\right)$ is a solution to (7) and (8). Arguing as above, but in reverse order with $t^{\prime}=T$, yields that

$$
\lambda_{t}^{*} V_{t}(c) \leq E_{t}\left\{\int_{t}^{T} \lambda_{s} F\left(c_{s}, \nu_{s}\right) d s\right\},
$$

which implies that $\left(\left\{V_{s}(c)\right\},\left\{\nu_{s}^{*}\right\}\right)$ solves the minimization problem in (9).

This lemma will be used in the next subsection to show the equivalence between recursive utility and variational utility, and also in our subsequent analysis.

\subsection{Characterization of Recursive Utility as Variational Utility}

Having extended Geoffard's formulation of variational utility to a stochastic setting in Section 2.1, we now show the equivalence between recursive utility and variational utility under uncertainty.

Let $f(c, v)$ be a (normalized) aggregator that is continuously differentiable, strictly concave, Lipschitz continuous in its second argument (with a Lipschitz coefficient less than $a$ ), and satisfies the linear growth condition in its first argument. ${ }^{7}$ Duffie and Epstein (1992) show that the (continuous-time) recursive utility process defined by $f$ exists and is given, for any $c \in D_{+}$, by the square-integrable semimartingale $\left\{V_{t}(c)\right\}$ that solves

$$
V_{t}=E_{t}\left[\int_{t}^{T} f\left(c_{s}, V_{s}\right) d s\right]
$$

Define a function $F$ by:

$$
F(c, \nu) \equiv \max _{u}[f(c, u)+\nu u]
$$

where $F$ is called the felicity function associated with the aggregator function $f$. Conversely, given any felicity function $F$, its associated aggregator $f$ is defined by:

$$
f(c, u)=\min _{\nu}[F(c, \nu)-u \nu] .
$$

Clearly, $f$ is the aggregator function associated with $F$ if and only if $F$ is the felicity function associated with $f$. The two functions are Legendre transforms of each other. The following theorem exploits this correspondence between aggregators and felicity functions to establish the equivalence between recursive utility and stochastic variational utility.

\footnotetext{
${ }^{7}$ See Duffie and Epstein (1992) for the definition of a normalized aggregator.
} 
Theorem 2.1 Suppose that $c \in D_{+}$. Let $f$ be a (normalized) aggregator that satisfies the conditions specified above and let $F$ be its associated felicity function. If $\left\{V_{t}(c)\right\}$ is the square-integrable semimartingale that solves (10), then there exists a discount rate process $\left\{\nu_{t}\right\}$ such that $\left(\left\{V_{t}(c)\right\},\left\{\nu_{t}\right\}\right)$ is the solution to the minimization problem (9); hence, $\left\{V_{t}(c)\right\}$ is the stochastic variational utility. Conversely, let $F$ be a felicity function and $f$ be its associated aggregator. If $\left(\left\{V_{t}(c)\right\},\left\{\nu_{t}\right\}\right)$ is the solution to problem (9), then $\left\{V_{t}(c)\right\}$ solves (10) and consequently is the recursive utility.

Proof: Suppose first that $f$ is an aggregator and $\left\{V_{t}(c)\right\}$ is the square-integrable semimartingale that solves (10). Let $F$ be the felicity function associated with $f$. Let $\nu \in \Pi$ be an arbitrary discount rate process and define

$$
\lambda_{t}=\exp \left\{\int_{0}^{t}-\nu_{s} d s\right\}
$$

Using first Itô's formula, and then (11), leads to:

$$
\lambda_{t} V_{t}(c)=E_{t}\left\{\int_{t}^{T} \lambda_{s}\left[f\left(c_{s}, V_{s}(c)\right)+\nu_{s} V_{s}(c)\right] d s\right\} \leq E_{t}\left\{\int_{t}^{T} \lambda_{s} F\left(c_{s}, \nu_{s}\right) d s\right\} .
$$

Thus,

$$
\lambda_{t} V_{t}(c) \leq \inf _{\nu \in \Pi} E_{t}\left\{\int_{t}^{T} \lambda_{s} F\left(c_{s}, \nu_{s}\right) d t\right\} .
$$

Choosing the particular $\nu_{t}$ to be

$$
\nu_{t}^{*}=-f_{V}\left(c_{t}, V_{t}(c)\right)
$$

we have

$$
F\left(c_{t}, \nu_{t}^{*}\right)=\max _{u}\left[f\left(c_{t}, u\right)+\nu_{t}^{*} u\right]=f\left(c_{t}, V_{t}\right)+\nu_{t}^{*} V_{t}
$$

Hence, $\left(\left\{V_{t}(c)\right\},\left\{\nu_{t}^{*}\right\}\right)$ is the solution to the minimization problem (9).

Conversely, let $\left(\left\{V_{t}(c)\right\},\left\{\nu_{t}\right\}\right)$ be the solution to the minimization problem (9). Again by Itô's formula,

$$
V_{t}(c)=V_{0}(c)+\int_{0}^{t} \nu_{s} V_{s}(c) d s-\int_{0}^{t} F\left(c_{s}, \nu_{s}\right) d s+\int_{0}^{t} \lambda_{s}^{-1} d M_{s}
$$

Using the aggregator $f$ associated with $F$ and Lemma 1, we have

$$
V_{t}(c)=V_{0}(c)-\int_{0}^{t} f\left(c_{s}, V_{s}(c)\right) d s+\int_{0}^{t} \lambda_{s}^{-1} d M_{s} .
$$


Thus, $\left\{V_{t}(c)\right\}$ solves (10). Since $f$ is automatically normalized when combined with the expected value certainty equivalent operator (Duffie and Epstein, 1992), $\left\{V_{t}(c)\right\}$ is the recursive utility.

Readers familiar with the recursive utility literature may have already noticed the similarity between the standard assumptions on the aggregator, $f$, and those that we impose on the felicity function, $F$. Thus, this seems to be an appropriate place to make some comments regarding the relationships between these assumptions. Also, in view of the above theorem, some comments on the properties of variational utility as related to those of recursive utility seem in order.

One of the assumptions Duffie and Epstein (1992) impose on $f$ in order to prove the existence of recursive utility is that $f$ is Lipschitz in its second argument. In light of (11) and (12), this corresponds to our Lipschitz assumption on F. Moreover, by Rockafellar (1970, Corollary 13.3.3), if $f(c, u)$ is concave in $u$, the Lipschitz property of $f$ necessarily leads to a bounded domain for the discount rate on which its associated felicity function $F$ is finite. Thus, our assumption that the discount rates be in $[-a, a]$ for some $a$ is, in a sense, not restrictive at all. By another result in Rockafellar (1970, Theorem 26.3), the strict convexity of the felicity function $F$ in $\nu$ follows from the assumption that its associated aggregator, $f$, is concave in $u$.

As the proof of Theorem 2.1 reveals, the equivalence between recursive utility and variational utility is based on the conjugate relation between the aggregator $f$ and the felicity function $F$. Thus, properties of recursive utility that can be derived from properties of the aggregator have counterparts in variational utility that can be derived from properties of the felicity function. Three properties seem to stand out as most useful: monotonicity, concavity, and the Inada property, a property that is often used to guarantee that optimal consumption is bounded away from zero. ${ }^{8}$ It follows immediately from (11) and (12) that the monotonicity of $f$ in $c$ implies the monotonicity of $F$ in $c$, and vice versa. The same holds for the Inada property. Concavity is a bit special. The concavity of a felicity function in $c$ corresponds to the concavity of its associated aggregator; the converse, however, is not true in general. The following is an example of how to use the relation between felicity functions and aggregators, along with Theorem 2.1, to derive properties of variational utility: since the concavity of an aggregator (in $c$ ) implies the concavity of recursive utility, the concavity of a felicity function (in $c$ ) implies the concavity of variational utility.

\footnotetext{
${ }^{8} \mathrm{An}$ aggregator $f(c, u)$ is said to have the Inada property if for all $c>0$, $\sup _{u}\left|f_{c}(c, u)\right|<\infty$, and $\lim _{c \rightarrow 0} \inf _{u}\left|f_{c}(c, u)\right|=\infty$. The Inada property for the felicity function, $F$, can be stated similarly.
} 


\subsection{Existence of Stochastic Variational Utility}

We turn now to the existence of variational utility. The existence theorem below is an easy consequence of Theorem 2.1.

Theorem 2.2 For any given $c \in D_{+}$, the minimization problem in (9) has a solution.

Proof: Let $f$ be the aggregator associated with the felicity function $F$. By the existence theorem in Duffie and Epstein (1992), there exists a semimartingale $\left\{V_{t}(c)\right\}$ that solves (10). Since $F$ is the felicity function associated with $f$, by Theorem 2.1, there exists a discount rate process $\left\{\nu_{t}\right\}$ such that $\left(\left\{V_{t}(c)\right\},\left\{\nu_{t}\right\}\right)$ solves $(9)$.

We have shown the equivalence between recursive utility and variational utility in a stochastic world, and thus the existence of stochastic variational utility. We conclude this section by describing how one can use dynamic programming to solve intertemporal problems when an individual agent has variational utility and the state variables are characterized by Markov processes.

\subsection{Variational Utility in a Markov Setting}

In many applications, uncertainty is assumed to be represented by a vector of Markov processes, so that one can use the ubiquitous dynamic programming technique to solve the optimization program. We now show that in a Markov setting the utility maximization problem with variational utility can be characterized as the solution to a Bellman equation, similar to that from the recursive utility literature. We will follow Duffie and Epstein (1992) closely in this subsection and hence will be brief. Readers are referred to that article for additional details.

For a given consumption process $c \in D_{+}$, let $X_{t}$ be a ( $m$-vector) state process such that

$$
d X_{t}=\eta\left(X_{t}, t, c_{t}\right) d t+\sigma\left(X_{t}, t, c_{t}\right) d B_{t},
$$

where $B$ is a $m$-dimensional Brownian motion. If, $c_{t}=c\left(X_{t}, t\right)$ for some measurable function $c$ and the diffusion and drift coefficients, $\sigma$ and $\eta$, satisfy the usual Lipschitz and linear growth conditions, then the stochastic differential equation in (13) has a unique solution. The differential operator $\mathcal{L}^{c}$ associated with the state process $X_{t}$, for any $c \in R_{+}$and $J \in C^{2,1}\left(R^{m+1},[0, T]\right)$, is given by

$$
\mathcal{L}^{c} J=J_{t}(x, t)+J_{x}(x, t) \eta(x, t, c)+\frac{1}{2} \operatorname{tr}\left[\sigma(x, t, c) \sigma(x, t, c)^{\top} J_{x x}(x, t)\right] .
$$


Let $\Gamma(x, t) \subset R$ be the "feasible set" given state $x$ at time $t$, in the sense that consumption $c_{t}$ must be chosen from the set $\Gamma(x, t)$. A process $c \in D_{+}$is admissible if, for all $t, c_{t} \in \Gamma\left(X_{t}, t\right)$. Let $D^{\Gamma}$ denote the set of admissible consumption processes. Then, the utility maximization problem for an agent with variational utility is

$$
\sup _{c \in D^{\Gamma}} V_{0}(c)
$$

An admissible consumption process $c^{*}$ is optimal if $V_{0}\left(c^{*}\right)=\sup _{c \in D^{\Gamma}} V_{0}(c)$. Let $J$ denote the value function of the utility maximization problem if there exists an optimal consumption process $c^{*}$ such that $J\left(X_{t}, \lambda_{t}, t\right)=\lambda_{t} V_{t}\left(c^{*}\right)$. We then have the following result.

Theorem 2.3 Suppose that $J$ is twice continuously differentiable in $x$, continuously differentiable in $\lambda$ and $t$, has a bounded derivative $J_{x}$ and that, for all $(x, \lambda, t)$

$$
\max _{c_{s} \in \Gamma(x, s)} \min _{\nu}\left[\mathcal{L}^{c} J(x, \lambda, t)+\lambda\left(F(c, \nu)-J_{\lambda} \nu\right)\right]=0
$$

with boundary condition $J(x, \lambda, T)=0$. Suppose, also, that there exists a solution $\left(C(x, t), \nu^{*}(x, t)\right)$ to the above Bellman equation. Finally, suppose that $X^{*}$ is the unique square-integrable process that solves (13) with $c_{t}$ replaced by $C\left(X_{t}^{*}, t\right)$. If $c_{t}^{*}=C\left(X_{t}^{*}, t\right)$ is square-integrable, then $J$ is the value function of

$$
\max _{c \in D^{\Gamma}} \min _{\nu} E_{t} \int_{t}^{T}\left[\lambda_{s} F\left(c_{s}, \nu_{s}\right)\right] d s
$$

subject to:

$$
d \lambda_{s}=-\nu_{s} \lambda_{s} d s, \quad \lambda_{t}=\lambda
$$

Proof: Suppose that $J$ satisfies the conditions of the theorem. Then,

$$
0=\min _{\nu}\left[\mathcal{L}^{c} J(x, 1, t)+\left(F(C(x, t), \nu)-J_{\lambda} \nu\right)\right]=\mathcal{L}^{c} J(x, 1, t)+f\left(C(x, t), J_{\lambda}\right),
$$

where $f$ is the aggregator associated with the felicity function $F$. By Duffie and Epstein (1992, Proposition 9$), J\left(X_{t}, 1, t\right)$ is the value function of the utility maximization problem with the recursive utility generated by the aggregator $f$; and, by Theorem 2.1 it is also the value function of our utility maximization problem with variational utility. Furthermore, from Lemma 1 we have that:

$$
J\left(X_{t}, 1, t\right)=V_{t}\left(c^{*}\right)=\min _{\nu_{s}} E_{t} \int_{t}^{T}\left[\exp \left(\int_{t}^{s}-\nu_{u} d u\right) F\left(C\left(X_{s}^{*}, s\right), \nu_{s}\right)\right] d s
$$


The claim of the theorem follows.

Remark: Notice that the solution to the Bellman equation is (positive) linear in $\lambda .{ }^{9}$ Thus the optimal consumption and discount rate do not depend on $\lambda$ and are written as: $\left(C(x, t), \nu^{*}(x, t)\right)$. Also, the boundedness assumption on $J_{x}$ can be relaxed; see Duffie and Epstein (1992).

We have now established the existence of stochastic variational utility, shown its equivalence to recursive utility, and described how one can use dynamic programming to solve the optimization problem of a single agent who has variational utility. In the rest of the paper, we analyze the problem of allocating resources efficiently over time in economies with multiple agents.

\section{$3 \quad$ Intertemporal Efficiency}

The importance of Pareto efficiency in both equilibrium analysis and asset pricing has been long recognized. See, for example, Negishi (1960) on equilibrium analysis, and Constantinides (1982) on asset pricing. The basic characterization of a Pareto-efficient allocation is the existence of an aggregate welfare function, or "representative agent" utility function, which can be constructed as a weighted sum of the individual utility functions (Duffie, 1996). While in theory one can prove, using a separation theorem, that these welfare weights exist, in applications the construction of an aggregate welfare function involves identifying a set of appropriate weights.

Constructing the appropriate set of weights is not easy. The difficulty arises from the fact that, even if each agent in the economy has recursive utility, the representative agent generally does not have recursive utility. In a dynamic setting, these weights will change over time and will in general depend on current time and the state of the world (Lucas and Stokey, 1984 and Kan,1995). The technique proposed by Lucas and Stokey ${ }^{10}$ is recursive but it involves a multiplicity of value functions ${ }^{11}$ and separate maximization and minimization problems. ${ }^{12}$ Thus, any technique that would help reduce the difficulty in the construction of the aggregate welfare function for a

\footnotetext{
${ }^{9}$ Hence $V_{t}\left(c^{*}\right)$ is the gradient of $J\left(X_{t}, \lambda_{t}, t\right)$ with respect to $\lambda_{t}$. This is trivial in the current context but the same property will hold in the case of multiple agents (Section 3 ).

${ }^{10}$ Lucas and Stokey applies the technique to discrete time problems, as does the generalized version by Dana and Le Van (1994). Epstein (1987) extends it to continuous time. Kan (1995) in discrete time and Duffie, Geoffard and Skiadas (1994) in continuous time extend it to uncertainty.

${ }^{11}$ Lucas and Stokey do obtain the one value function corresponding to aggregate welfare (the function $v(k, \theta)$ in their notation) but their algorithm also includes the explicit determination of the gamut of individual investors' utility levels (the vector $z$ in their notation), in addition to the need, which we also have, to obtain the individual investors' time varying weights (the vector $w$ for next period, $\theta$ for this period, in their notation).

${ }^{12}$ Because of the recursive structure, Kan (1995) refers to the technique as "dynamic programming" but it is a vectorial form of dynamic programming.
} 
Pareto-efficient allocation is valuable. The main objective of this section is to show in a dynamic setting the usefulness of variational utility in this regard. We show that the standard form of dynamic programming, involving one value (aggregate welfare) function, can still be used. The non-recursivity of the welfare function is handled by means of appropriate state variables (which are the time varying weights). These would have been present anyway in the Lucas and Stokey program applied to the case of uncertainty. ${ }^{13}$ What we are proposing is indeed a net reduction in the complexity of the formulation.

The definition of efficiency that we will use is the standard one used by Epstein (1987) and Duffie, Geoffard and Skiadas (1994). There are $N$ agents in the economy, each characterized by a variational utility process $\left\{V_{t}^{n}\right\}, n=1, \ldots, N$. In light of Theorem 2.1, this is equivalent to assuming that the agents' preferences are represented by recursive utility functions. Let $F^{n}$ and $f^{n}, n=1, \ldots, N$, denote the corresponding felicity functions and aggregators respectively. There is a total endowment $e$, an element of $D_{+}$, that is to be shared among the $N$ agents. An allocation $c=\left(c^{1}, \ldots, c^{N}\right) \in D_{+}^{N}$ is feasible if $e_{t} \geq \sum_{n=1}^{N} c_{t}^{n}$. Let $\alpha=\left(\alpha^{1}, \ldots, \alpha^{N}\right)$ be a $N$ vector of strictly positive numbers. An allocation is said to be $\alpha$-efficient if it is feasible and it maximizes $\sum_{n=1}^{N} \alpha^{n} V_{0}^{n}\left(c^{n}\right)$ among all feasible allocations. In what follows, any symbol which carries a superscript $n(n=1, \ldots, N)$, refers to a variable attached to a particular agent of the economy. When that same symbol appears without the superscript $n$, it is understood to refer to the collection of variables of the whole population of the economy. This applies to $c, \alpha, \nu, V, \lambda, f$, and $F$.

In the first subsection below, we illustrate the basic idea of constructing the aggregate welfare function in an economy without uncertainty. Then, in the following subsection, we provide a more rigorous characterization of this idea under uncertainty.

\subsection{The Certainty Case}

We start with Epstein's (1987) characterization of $\alpha$-efficient allocations:

$$
\max _{\left\{c_{s}^{n}\right\}} \int_{s \geq t} \sum_{n=1}^{N}\left[\lambda_{s}^{n} f^{n}\left(c_{s}^{n}, V_{s}^{n}\right)-\dot{\lambda}_{s}^{n} V_{s}^{n}\right] d s
$$

\footnotetext{
${ }^{13}$ See below, in Section 3.2, the Duffie, Geoffard, Skiadas (1994) formulation.
} 
subject to:

$$
\begin{aligned}
\sum_{n=1}^{N} c_{s}^{n} & \leq e\left(x_{s}\right), \\
\dot{V}_{s}^{n} & =-f^{n}\left(c_{s}^{n}, V_{s}^{n}\right) ; n=1, \ldots, N ; \\
\dot{\lambda}_{s}^{n} & =\lambda^{n} f_{V}^{n}\left(c_{s}^{n}, V_{s}^{n}\right) ; \lambda_{0}^{n}=\alpha^{n} ; n=1, \ldots, N ; \\
\dot{x}_{s} & =\eta\left(x_{s}\right),
\end{aligned}
$$

where a dot over a symbol denotes the time derivative and $\lambda^{n}, V^{n}$ and $x$ are viewed as state variables. The cumbersome aspect of this formulation is that it involves simultaneously the time paths of both $\lambda^{n}$ and $V^{n}$ for all $n$ and hence a multiplicity of unknown value functions.

We illustrate now that the following optimization problem, with a suitable definition of the felicity functions, $F^{n}\left(c^{n}, \nu^{n}\right)$, has a solution in common with the Epstein problem:

$$
J(x, \lambda) \equiv \max _{\left\{c_{s}^{n}\right\}} \min _{\left\{\nu_{s}^{n}\right\}} \int_{s \geq t} \sum_{n=1}^{N}\left[\lambda_{s}^{n} F^{n}\left(c_{s}^{n}, \nu_{s}^{n}\right)\right] d s
$$

subject to (16), (19), and:

$$
\dot{\lambda}_{s}^{n}=-\nu_{s}^{n} \lambda_{s}^{n} ; n=1, \ldots, N
$$

where $\lambda=\left(\lambda^{1}, \ldots, \lambda^{N}\right)$. The Bellman equation for this problem is:

$$
0=\max _{\left\{c^{n}\right\}}\left\{\sum_{n=1}^{N} \lambda^{n} \min _{\nu^{n}}\left[F^{n}\left(c^{n}, \nu^{n}\right)-J_{\lambda^{n}}(x, \lambda) \nu^{n}\right]+J_{x}(x, \lambda) b(x)\right\} .
$$

Differentiate it with respect to each $\lambda^{n}$ to get:

$$
0=\min _{\nu^{n}}\left[F^{n}\left(c^{n}, \nu^{n}\right)-J_{\lambda^{n}}(x, \lambda) \nu^{n}\right]-\sum_{j=1}^{N} \lambda^{j} J_{\lambda^{n} \lambda^{j}}(x, \lambda) \nu^{j}(t)+J_{\lambda^{n}, x}(x, \lambda) \eta(x) .
$$

Now, define the functions $V^{n}$ :

$$
V^{n}(x, \lambda) \equiv J_{\lambda^{n}}(x, \lambda),
$$

with implied drift:

$$
\dot{V}_{s}^{n}=J_{\lambda^{n}, x}(x, \lambda) \eta\left(x_{s}\right)-\sum_{j=1}^{N} \lambda_{s}^{j} J_{\lambda^{n} \lambda^{j}}(x, \lambda) \nu_{s}^{j}
$$


and also define the aggregator functions, $f^{n}$, associated with $F^{n}$ :

$$
f^{n}\left(c^{n}, u\right) \equiv \min _{\nu}\left[F^{n}\left(c^{n}, \nu\right)-u \nu\right]
$$

Then, (21) may be rewritten as

$$
0=f^{n}\left(c^{n}(t), V^{n}\right)+\dot{V}^{n},
$$

which is (17), the second constraint in Epstein's characterization of $\alpha$-efficient allocations. As for (20), it becomes, on the basis of (11):

$$
\max _{\left\{c_{s}^{n}\right\}} \int_{s \geq t} \sum_{n=1}^{N}\left(\lambda_{s}^{n} \max _{u}\left[f^{n}\left(c_{s}^{n}, u\right)+\nu u\right]\right) d s=\max _{\left\{c_{s}^{n}\right\}} \int_{s \geq t} \sum_{n=1}^{N}\left(\lambda_{s}^{n} \max _{u}\left[f^{n}\left(c_{s}^{n}, u\right)-\frac{\dot{\lambda}^{n}}{\lambda^{n}} u\right]\right) d s .
$$

One recognizes Epstein's objective function, equation (15). As for Epstein's third constraint, equation (18), it is the first-order condition of the $\max _{u}$ problem above.

Thus, by formulating the $\alpha$-efficient allocation problem by means of (20), we have reduced the number of unknown value functions to only one. ${ }^{14}$.

\subsection{The Uncertainty Case}

Duffie, Geoffard and Skiadas (1994) show that, under the condition that $f^{n}, n=1, \ldots, N$, are concave, $\alpha$-efficient allocations always exist. ${ }^{15}$ They show further ${ }^{16}$ that if an $\alpha$-efficient allocation is bounded away from zero, which is the case when the total endowment $e$ is bounded away from zero and $f^{n}$ have the Inada property, then there exists a vector of time-varying weights $\lambda$ and a vector of time-varying values $V$ such that an $\alpha$-efficient allocation can be characterized as:

$$
\max _{c_{s}^{n}} \sum_{n=1}^{N} \lambda_{t}^{n} f^{n}\left(c_{t}^{n}, V_{t}^{n}\right)
$$

subject to the feasibility constraint and the following double set of equations:

$$
\begin{aligned}
\lambda_{t}^{n} & =\alpha^{n} \exp \left(\int_{0}^{t} f_{V}^{n}\left(c_{s}^{n}, V_{s}^{n}\right) d s\right) ; n=1, \ldots, N, \\
V_{t}^{n} & =E_{t}\left[\int_{t}^{T} f\left(c_{s}^{n}, V_{s}^{n}\right) d s\right] ; n=1, \ldots, N .
\end{aligned}
$$

\footnotetext{
${ }^{14}$ Moreover, the value function is positively linearly homogeneous in $\lambda$, which is a property that can be exploited in specific applications; one such application is presented in Section 4.

${ }^{15}$ See their Theorem 1.

${ }^{16}$ See their Propositions 4 and 5.
} 
Moreover, if $f^{n}$ are strictly concave in $c$ and three-times continuously differentiable, then the $\alpha$ efficient allocation is unique.

We relate now $\alpha$-efficient allocations to an optimization problem that will prove analytically more tractable. Assume that $F(c, \nu)$ is strictly concave in $c$. The alternative optimization problem we shall consider is

$$
J(x, \lambda, t) \equiv \max _{\left\{c_{s}^{n}\right\}} \min _{\left\{\nu_{s}^{n}\right\}} E_{t}\left\{\int_{t}^{T} \sum_{n=1}^{N} \lambda_{s}^{n} F^{n}\left(c_{s}^{n}, \nu_{s}^{n}\right) d s\right\}
$$

subject to

$$
\begin{aligned}
\sum_{n=1}^{N} c_{s}^{n} & \leq e\left(x_{s}\right), \\
d \lambda_{s}^{n} & =-\nu_{s}^{n} \lambda_{s}^{n} d s ; \quad \lambda_{0}^{n}=\alpha^{n} ; n=1, \ldots, N, \\
J(x, \lambda, T) & =0 .
\end{aligned}
$$

In light of Theorem 2.1, and with $\alpha \equiv\left(\alpha^{1}, \ldots, \alpha^{N}\right)$ :

$$
J(x, \alpha, 0)=\max _{\left\{c_{s}^{n}\right\}} \sum_{n=1}^{N} \alpha^{n} V_{0}^{n}\left(c^{n}\right) .
$$

Since the existence of $\alpha$-efficient allocations solutions to (22)-(24) is guaranteed under the conditions mentioned above (Duffie, Geoffard and Skiadas, 1994), we proceed to the characterization of these allocations using the above optimization problem and show that this problem is equivalent to the problem given by (22)-(24).

We assume that the ( $m$-vector) state process $X_{t}$ is the solution of the stochastic differential equation in (13) and its associated differential operator is given in (14). The Bellman equation of (25) is, by definition,

$$
0=\max _{\left\{c_{s}^{n}\right\}} \min _{\left\{\nu_{s}^{n}\right\}} \sum_{n=1}^{N} \lambda^{n}\left[F^{n}\left(c^{n}, \nu^{n}\right)-J_{\lambda^{n}}(x, \lambda, t) \nu^{n}\right]+\mathcal{L} J(x, \lambda, t) .
$$

Remark: It is clear by inspection that this Bellman equation admits a solution which is positively linearly homogeneous in $\lambda$.

We establish first that the optimization problem in (25) can be characterized by the Bellman equation (28). 
Theorem 3.1 Suppose that (25) has a solution, that its value function $J$ is twice continuously differentiable in $x$, continuously differentiable in $\lambda$ and $t$ and has bounded derivatives, $J_{\lambda}$ and $J_{x}$, and that $J$ satisfies the boundary condition $J(x, \lambda, T)=0$.

(a) If there exists a solution $\left(C(x, \lambda, t), \nu^{*}(x, \lambda, t)\right)$ to the Bellman equation in (28), and if $c_{t}^{*}=$ $C\left(X_{t}, \lambda_{t}^{*}, t\right)$ is square-integrable, where $\left\{\lambda_{t}^{*}\right\}$ is the discount factor process associated with $\nu_{t}^{*}$, then $J$ is the value function in (25).

(b) Conversely, if $J$ is the value function in (25) and $\left(c_{t}^{*}, \nu_{t}^{*}, \lambda_{t}^{*}\right)$, with $\left\{\lambda_{t}^{*}\right\}$ as in part (a), solves the problem in (25), then they satisfy the Bellman equation (28).

Proof: For (a), by the Implicit Function Theorem, there exists for each $n$ a continuously differentiable function $\nu^{n}(c, V)$ that solves the minimization problem in the Minimum Principle. That is,

$$
F^{n}\left(c, \nu^{n}(c, V)\right)-\nu^{n}(c, V) V=\min _{\nu^{n}}\left[F^{n}\left(c, \nu^{n}\right)-\nu^{n} V\right]
$$

Since $\left(C(x, \lambda, t), \nu^{*}(x, \lambda, t)\right)$ solves the Bellman equation (28),

$$
\nu^{* n}(x, \lambda, t)=\nu^{n}\left(C(x, \lambda, t), J_{\lambda^{n}}(x, \lambda, t)\right) .
$$

Let $c$ be an arbitrary feasible allocation; it follows from the Bellman equation that

$$
0 \geq \sum_{n=1}^{N} \lambda^{n}\left[F^{n}\left(c^{n}, \nu^{n}\left(c^{n}, J_{\lambda^{n}}\right)\right)-J_{\lambda^{n}}(x, \lambda, t) \nu^{n}\left(c^{n}, J_{\lambda^{n}}\right)\right]+\mathcal{L} J(x, \lambda, t) .
$$

Then, the standard argument for verification theorems (Fleming and Rishel, 1975) leads to

$$
J(x, \lambda, t) \geq E_{t}\left\{\int_{t}^{T} \sum_{n=1}^{N} \lambda_{s}^{n} F^{n}\left(c_{s}^{n}, \nu_{s}^{n}\left(c^{n}, J_{\lambda^{n}}\right)\right) d s\right\} \geq \min _{\left\{\nu_{s}^{n}\right\}} E_{t}\left\{\int_{t}^{T} \sum_{n=1}^{N} \lambda_{s}^{n} F^{n}\left(c_{s}^{n}, \nu_{s}^{n}\right) d s\right\}
$$

Thus,

$$
J(x, \lambda, t) \geq \max _{\left\{c_{s}^{n}\right\}\left\{\nu_{s}^{n}\right\}} E_{t}\left\{\int_{t}^{T} \sum_{n=1}^{N} \lambda_{s}^{n} F^{n}\left(c_{s}^{n}, \nu_{s}^{n}\right) d s\right\} .
$$

Equality holds when the arbitrary $c$ is replaced by $c_{t}^{*}$. 
For (b), fix $\lambda_{t}^{*}$ and define

$$
\hat{J}(x, \lambda, t)=\min _{\left\{\nu_{s}^{n}\right\}} E_{t}\left\{\int_{t}^{T} \sum_{n=1}^{N} \lambda_{s}^{n} F^{n}\left(c_{s}^{* n}, \nu_{s}^{n}\right) d s\right\}
$$

subject to (27). Then $J(x, \lambda, t) \geq \hat{J}(x, \lambda, t)$ and $J\left(x, \lambda_{t}^{*}, t\right)=\hat{J}\left(x, \lambda_{t}^{*}, t\right)$. It can be verified that both $J(x, \lambda, t)$ and $\hat{J}(x, \lambda, t)$ are convex in $\lambda$. Thus, by Lucas and Stokey (1989, p.84):

$$
J_{\lambda^{n}}\left(X_{t}, \lambda^{*}, t\right)=\hat{J}_{\lambda^{n}}\left(X_{t}, \lambda^{*}, t\right)=\min _{\left\{\nu_{s}^{n}\right\}} E_{t}\left\{\int_{t}^{T} \lambda_{s}^{n} F^{n}\left(c_{s}^{* n}, \nu_{s}^{n}\right) d s\right\} .
$$

Then, by Lemma 1 and the Implicit Function Theorem, $\nu_{t}^{* n}=\nu^{n}\left(c_{s}^{* n}, J_{\lambda^{n}}\left(X_{t}, \lambda_{t}^{*}, t\right)\right)$, as in the proof of part (a). In particular, $\left\{\nu_{t}^{*}\right\}$ has right continuous sample paths.

Now, let $c$ be a feasible allocation such that

$$
\lambda_{t}^{n} V_{t}^{n}\left(c^{n}\right)=\inf _{\nu_{s}^{n}} E_{t}\left\{\int_{t}^{t^{\prime}} \lambda_{s}^{n} F^{n}\left(c^{n}, \nu_{s}^{n}\right) d s+\lambda_{t^{\prime}}^{n} J_{\lambda^{n}}\left(X_{t}, \lambda_{t^{\prime}}, t\right)\right\}
$$

has a solution $\left(\left\{V_{s}^{n}\left(c^{n}\right)\right\},\left\{\nu_{s}^{n}\right\}\right)$. It follows from Lemma 1 that $\nu_{s}^{n}=\nu^{n}\left(c^{n}, V_{s}^{n}\left(c^{n}\right)\right)$. Since

$$
J(x, \lambda, t) \geq E_{t}\left\{\int_{t}^{t^{\prime}} \sum_{n=1}^{N} \lambda_{s}^{n} F^{n}\left(c_{s}^{n}, \nu^{n}\left(c^{n}, V_{s}^{n}\left(c^{n}\right)\right) d s+J\left(X_{t^{\prime}}, \lambda_{t^{\prime}}, t^{\prime}\right)\right\}\right.
$$

where we have used the fact that $J$ is linearly homogeneous in $\lambda$, by Itô's formula:

$$
0 \geq E_{t}\left\{\int_{t}^{t^{\prime}}\left[\sum_{n=1}^{N} \lambda_{s}^{n}\left(F^{n}\left[c_{s}^{n}, \nu^{n}\left(c_{s}^{n}, V_{s}^{n}\left(c^{n}\right)\right)\right]-\nu_{s}^{n} J_{\lambda^{n}}\right)+\mathcal{L} J\right] d s\right\}
$$

Taking limits, and noting the right continuity of the integrand, we have

$$
0 \geq \sum_{n=1}^{N} \lambda_{t}^{n}\left(F^{n}\left[c_{t}^{n}, \nu^{n}\left(c_{t}^{n}, J_{\lambda^{n}}\left(x, \lambda_{t}, t\right)\right)\right]-\nu_{t}^{n} J_{\lambda^{n}}\right)+\mathcal{L} J .
$$

Thus, given that $c_{t}$ is arbitrary,

$$
0 \geq \max _{c^{n}} \min _{\nu^{n}} \sum_{n=1}^{N} \lambda_{t}^{n}\left(F^{n}\left(c^{n}, \nu^{n}\right)-\nu^{n} J_{\lambda^{n}}\right)+\mathcal{L} J .
$$


Finally, since at $c_{t}^{*}$ we can replace the inequalities in the above derivation with equalities, we have that $\left(J, c_{t}^{*}, \nu_{t}^{*}, \lambda_{t}^{*}\right)$ satisfies the Bellman equation.

Now we are ready to characterize $\alpha$-efficient allocations as solutions of the Bellman equation (28).

Theorem 3.2 The problem in (22), (23) and (24) has a solution if and only if the Bellman equation (28) has a solution.

Proof: Suppose first that $J(x, \lambda, t), C(x, \lambda, t)$, and $\nu(x, \lambda, t)$ taken together is a solution to (28). Substitute them into (28), and differentiate with respect to each $\lambda^{n}$, to get

$$
\begin{aligned}
0 & =F^{n}\left(C^{n}, \nu^{n}\right)-J_{\lambda^{n}} \nu^{n}-\sum_{j=1}^{N} \nu^{j} \lambda^{j} J_{\lambda^{j} \lambda^{n}}(x, \lambda, t)+\mathcal{L} J_{\lambda^{n}}(x, \lambda, t) \\
& =f^{n}\left(C^{n}, J_{\lambda^{n}}\right)-\sum_{j=1}^{N} \nu^{j} \lambda^{j} J_{\lambda^{j} \lambda^{n}}(x, \lambda, t)+\mathcal{L} J_{\lambda^{n}}(x, \lambda, t) .
\end{aligned}
$$

It follows from Duffie and Epstein (1992, Proposition 9), with $(x, \lambda)$ as the state variables and $\left\{C^{n}(x, \lambda, t)\right\}$ as their $\Gamma(x, \lambda, t)$, that $V_{t}^{n}\left(C^{n}\right)=J_{\lambda^{n}}$ and that $V_{t}^{n}\left(C^{n}\right)$ satisfies (24). Furthermore, it follows from the minimization problem in the Bellman equation (28), from equation (12), and from the Envelope Theorem, that $\lambda_{t}^{n}$ satisfies (23). Finally, upon using (12) again, the Bellman equation (28) leads to

$$
0=\max _{c^{n}} \sum_{n=1}^{N} \lambda_{t}^{n} f^{n}\left(c^{n}, J_{\lambda^{n}}\right)+\mathcal{L} J
$$

Thus, $C^{n}(x, \lambda, t)$ solves

$$
\max _{c^{n}} \sum_{n=1}^{N} \lambda_{t}^{n} f^{n}\left(c^{n}, J_{\lambda^{n}}\right)
$$

which is $(22)$.

Conversely, suppose that $\left(c_{t}^{*}, V_{t}^{*}, \lambda_{t}^{*}\right)$ satisfy (22), (23) and (24). Let $\nu_{t}^{* n}=-f_{V}\left(c_{t}^{* n}, V_{t}^{* n}\right)$. Then

$$
d \lambda_{t}^{* n}=-\nu_{t}^{* n} \lambda_{t}^{* n} d t
$$


Since by Theorem 2.1,

$$
\sum_{n=1}^{N} \lambda_{t}^{* n} V_{t}^{* n}=E_{t}\left\{\int_{t}^{T} \sum_{n=1}^{N}\left[\lambda_{s}^{* n} F^{n}\left(c_{s}^{* n}, \nu_{s}^{* n}\right)\right] d s\right\} \leq J\left(X_{t}, \lambda_{t}^{*}, t\right)
$$

the allocation must be a solution to (25). Thus, from Theorem 3.1, $\left(c_{t}^{*}, \nu_{t}^{*}, \lambda_{t}^{*}, J\right)$ satisfies the Bellman equation (28).

An immediate consequence of Theorems 3.1 and 3.2 is that, given recursive utilities $\left\{V_{t}^{n}\right\}$, a feasible allocation $c^{*}$ is $\alpha$-efficient if and only if there exists a vector of dynamic weight processes, $\lambda_{t}^{*}=\left(\lambda_{t}^{* 1}, \ldots, \lambda_{t}^{* N}\right)$, such that $\lambda_{0}^{*}=\alpha$ and the allocation maximizes

$$
\sum_{n=1}^{N} \lambda_{t}^{* n} V_{t}^{n}\left(c^{n}\right), \forall t
$$

Furthermore, the process for the weights is given by

$$
d \lambda_{t}^{* n}=-\nu_{t}^{* n} \lambda_{t}^{* n} d t, \quad \lambda_{0}^{*}=\alpha ; \quad n=1, \ldots, N,
$$

where $\nu_{t}^{*}$ is part of the solution to the Bellman equation (28).

Our characterization of $\alpha$-efficient allocations as the solution to the Bellman equation (28) is closely related to the max-min characterization given by Lucas and Stokey (1984) and Kan (1995). The difference is that we are able to combine their separate max and min problems into a single max-min problem with one value function. The individual investors' utility level remain implicit, encapsulated inside the welfare function; at no point in the course of our method do we need to determine them explicitly. The relationship between the aggregate welfare process and the individual investors' utility processes has been established in the course of the proof: $V_{t}^{n}\left(C^{n}\right)=J_{\lambda^{n}}$. The latter are the gradients of the former taken with respect to the weights. By virtue of Euler's theorem, the aggregate welfare is indeed equal to a weighted sum of individual utility levels but the weights are variable, which is how the Pareto-optimality problem is generalized from time-additive utilities to recursive utilities.

\section{An Example}

In this section, we use a specific example to illustrate how one can use variational utility to solve problems in multi-agent economies. We also discuss the advantages of our method compared to that proposed in Duffie, Geoffard and Skiadas (1994). 
In our example, we consider an economy with $n=1, \ldots, N \geq$ agents. The preferences of each consumer are given by a special case of recursive utility, sometimes called Kreps-Porteus utility. This utility function exhibits constant elasticity in two dimensions: risk aversion and intertemporal substitution. The constant degree of relative risk aversion is equal to $1-\gamma$, with $\gamma<1, \neq 0$. Separately, the elasticity of intertemporal substitution, different for each investor is equal to $1 /\left(1-\rho^{n}\right)$, where $\rho^{n}<1, \neq 0 .{ }^{17}$ We also assume that each consumer has a different rate of impatience, $\beta^{n}$. Thus, the felicity functions, $F^{n}\left(c^{n}, \nu^{n}\right)$, are: ${ }^{18}$

$$
F^{n}\left(c^{n}, \nu^{n}\right)=\beta^{n} \frac{\left(c^{n}\right)^{\gamma}}{\gamma}\left[-\frac{\rho^{n}-\gamma}{\gamma-\frac{\rho^{n} \nu^{n}}{\beta^{n}}}\right]^{\frac{\gamma}{\rho^{n}}-1}
$$

The investors consume a single good and have access to two investment opportunities: (1) they can buy shares in a constant-returns-to-scale production activity, whose random output per unit of capital has a constant Gaussian distribution with fixed drift and diffusion parameters $\eta$ and $\sigma$; and, (2) they can borrow and lend to and from each other at the equilibrium riskless rate $r_{t}$, which varies over time in an endogenous fashion. Other notations are as follows:

$W^{n}$ : wealth of each investor;

$S=\sum_{n=1}^{N} W^{n}$ : aggregate wealth and capital stock; ${ }^{19}$

$w^{n}$ : share of each investor's wealth invested in the risky production opportunity;

$c^{n}$ : consumption rate of each investor.

The dynamics of an investor's wealth, for a given investment decision $w^{n}$ and a given consumption decision $c$, are well known:

$$
d W_{s}^{n}=\left\{W_{s}^{n}\left[r_{s}+w_{s}^{n}\left(\eta-r_{s}\right)\right]-c_{s}^{n}\right\} d s+W_{s}^{n} w_{s}^{n} \sigma d B_{s} ; n=1, \ldots, N
$$

where $B$ is the one-dimensional Brownian motion affecting production. The dynamics of the aggregate capital stock is the sum of the wealth equations ( with $\sum_{n=1}^{N} w^{n}=1$ ). It simply reflects the flow of goods; see equation (30) below.

Geoffard (1995) and Section 2 above allow us to write the objective function of each investor-

\footnotetext{
${ }^{17}$ The special case of the time-additive expected lifetime utility function is obtained for $\gamma=\rho$.

18 The felicity function (29) is the Legendre transform of the (normalized) aggregator proposed by Duffie and Epstein (1992, page 367) for isoelastic, Kreps-Porteus preferences: $f(c, v)=\frac{\beta}{\rho} \frac{c^{\rho}-(\gamma v)^{\rho / \gamma}}{(\gamma v)^{\frac{\rho}{\gamma}}-1}$.

19 take the same value because of the assumption of constant returns to scale.
} 
consumer with the aforementioned preferences in a pseudo time-additive form:

$$
\begin{aligned}
\max _{c^{n}} \min _{\nu^{n}} & E_{t}\left\{\int_{t}^{\infty} \lambda_{s}^{n} F^{n}\left(c_{s}^{n}, \nu_{s}^{n}\right) d s\right\}, \\
\text { subject to : } & d \lambda_{s}^{n}=-\nu_{s}^{n} \lambda_{s}^{n} d s,
\end{aligned}
$$

where $\lambda_{s}$ may be interpreted as a psychological discount factor applicable to utility. However, the discount factor is endogenous, since its dynamics are governed by the "choice variable" $\nu^{n}$.

The objective function of the central planner is the sum of the individual objective functions; hence, it may be written as:

$$
J\left(S, \lambda^{1}, \ldots, \lambda^{N}\right) \equiv \max _{\left\{c^{n}\right\}} \min _{\left\{\nu^{n}\right\}} E_{t}\left\{\int_{t}^{\infty}\left[\sum_{n=1}^{N} \lambda_{s}^{n} F^{n}\left(c_{s}^{n}, \nu_{s}^{n}\right)\right] d s\right\}
$$

subject to:

$$
\begin{aligned}
& d S_{s}=\left(S_{s}\left[w_{s}\left(\eta-r_{s}\right)+r_{s}\right]-\sum_{n=1}^{N} c_{s}^{n}\right) d s+\sigma w_{s} S_{s} d B_{s}, S(0)=S, \\
& d \lambda_{s}^{n}=-\nu_{s}^{n} \lambda_{s}^{n} d s, \quad \lambda_{0}^{n}=\alpha^{n} ; \quad n=1, \ldots, N
\end{aligned}
$$

where $\alpha^{n}$ are some strictly positive constants and $w_{s}$ is the portfolio weight on the risky asset. The value function $J\left(S, \lambda^{1}, \ldots, \lambda^{N}\right)$ satisfies the Bellman equation, which is:

$0=\max _{\left\{c^{n}, w\right\}} \min _{\left\{\nu^{n}\right\}}\left[\sum_{n=1}^{N} \lambda^{n}\left(F^{n}\left(c^{n}, \nu^{n}\right)-J_{\lambda^{n}} \nu^{n}\right)+J_{S}\left(S\left[w\left(\eta-r_{t}\right)+r_{t}\right]-\sum_{n=1}^{N} c^{n}\right)+\frac{1}{2} J_{S S}(\sigma)^{2}(w S)^{2}\right]$.

After substituting the optimal values for $\nu^{n}$, one obtains:

$$
0=\max _{\left\{c^{n}, w\right\}}\left[\sum_{n=1}^{N} \lambda^{n} \frac{\beta^{n}}{\rho^{n}} \frac{\left(c^{n}\right)^{\rho^{n}}-\left(\gamma J_{\lambda^{n}}\right)^{\rho^{n} / \gamma}}{\left(\gamma J_{\lambda^{n}}\right)^{\frac{\rho^{n}}{\gamma}-1}}+J_{S}\left(S\left[w\left(\eta-r_{s}\right)+r_{s}\right]-\sum_{n=1}^{N} c^{n}\right)+\frac{1}{2} J_{S S} \sigma^{2}(w S)^{2}\right] .
$$

Thus, to obtain the efficient allocation one needs to solve for only the single value function, $J\left(S, \lambda^{1}, \ldots, \lambda^{N}\right)$, after substituting the first-order conditions:

$$
\begin{aligned}
0 & =\left(\eta-r_{t}\right) J_{S}+J_{S S} w^{*} \sigma^{2} S \\
J_{S} & =\lambda^{n} \frac{\beta^{n}\left(c^{n}\right)^{\rho^{n}-1}}{\left(\gamma J_{\lambda^{n}}\right)^{\frac{\rho^{n}}{\gamma}-1}} ; n=1, \ldots, N .
\end{aligned}
$$


This characterization of efficient allocation in terms of the single value function in (31) can be compared with that of Duffie, Geoffard and Skiadas (1994), which in this example would be the following set of double equations: ${ }^{20}$

$$
\begin{aligned}
\lambda_{t}^{n} & =\alpha^{n} \exp \left\{\int_{0}^{t} \frac{\beta^{n}}{\rho^{n}}\left[\left(1-\frac{\rho^{n}}{\gamma}\right)\left(c_{s}^{n}\right)^{\rho^{n}}\left(\gamma V_{s}^{n}\right)^{-\frac{\rho^{n}}{\gamma}}-\gamma\right] d s\right\} ; n=1, \ldots, N, \\
V_{t}^{n} & =E_{t} \int_{t}^{T} \frac{\beta^{n}}{\rho^{n}} \frac{\left(c_{s}^{n}\right)^{\rho^{n}}-\left(\gamma V_{s}^{n}\right)^{\rho^{n} / \gamma}}{\left(\gamma V_{s}^{n}\right)^{\frac{\rho^{n}}{\gamma}-1}} d s ; n=1, \ldots, N
\end{aligned}
$$

a far more complex system of $N$ forward and $N$ backward stochastic integral equations. The difficulty in solving such forward-backward equations is discussed in Schroder and Skiadas (1997).

Duffie et al. do not suggest any method to solve the above system but the following can be envisaged. ${ }^{21}$ Introduce the unknown functions $V^{n}\left(S, \lambda^{1}, \ldots, \lambda^{N}\right)$. Equation (35) means that the drift of $V_{t}^{n}$ is equal to:

$$
-\frac{\beta}{\rho^{n}} \frac{\left(c_{s}^{n}\right)^{\rho^{n}}-\left(\alpha V_{s}^{n}\right)^{\rho^{n} / \gamma}}{\left(\gamma V_{s}^{n}\right)^{\frac{\rho^{n}}{\gamma}-1}},
$$

while (34) means that the drift of $\lambda_{t}^{n}$ is equal to:

$$
\frac{\beta^{n}}{\rho^{n}}\left[\left(1-\frac{\rho^{n}}{\gamma}\right)\left(c_{s}^{n}\right)^{\rho^{n}}\left(\gamma V_{s}^{n}\right)^{-\frac{\rho^{n}}{\gamma}}-\gamma\right] \lambda_{s}^{n}
$$

Assembling this information into one partial differential equation for each agent, we get:

$$
\begin{aligned}
0= & \frac{\beta}{\rho^{n}} \frac{\left(c_{s}^{n}\right)^{\rho^{n}}-\left(\gamma V_{s}^{n}\right)^{\rho^{n} / \gamma}}{\left(\gamma V_{s}^{n}\right)^{\frac{\rho^{n}}{\gamma}-1}}+V_{S}^{n}\left(\eta S-\sum_{n=1}^{N} c^{n}\right)+\frac{1}{2} V_{S S}^{n}(\sigma)^{2}(S)^{2} \\
& +\sum_{j=1,2} V_{\lambda^{j}}^{n} \frac{\beta^{j}}{\rho^{j}}\left[\left(1-\frac{\rho^{n}}{\gamma}\right)\left(c^{j}\right)^{\rho^{j}}\left(\gamma V^{j}\right)^{-\frac{\rho^{j}}{\gamma}}-\gamma\right] \lambda^{j} ; n=1, \ldots, N,
\end{aligned}
$$

where we have imposed the market-clearing condition that $w=1$ in equilibrium. To this system of partial differential equations, one must append the first-order condition for consumptions that, from (22), is:

$$
\lambda^{i} \frac{\beta^{i}\left(c^{i}\right)^{\rho^{i}-1}}{\left(\gamma V^{i}\right)^{\frac{\rho^{i}}{\gamma}-1}}=\lambda^{j} \frac{\beta^{j}\left(c^{j}\right)^{\rho^{j}-1}}{\left(\gamma V^{j}\right)^{\frac{\rho^{j}}{\gamma}-1}} .
$$

\footnotetext{
${ }^{20}$ See $(23)$ and (24) above.

${ }^{21}$ Schroder and Skiadas (1997) also discuss, for the case of a single agent, how one can use the results in Ma, Protter and Yong (1994) to simplify the forward-backward stochastic differential equations.
} 
Compared to the $2 \times N$ unknown functions to be identified in (34) and (35), we now have only $N$ partial differential equations to solve. However, even this system of $N$ partial differential equations, with the cross-equation restriction in (37), will typically be more difficult to solve than the single differential equation in (31), derived using variational utility. Below, we show how one can use the single differential equation in (31) to obtain an explicit solution for the economy considered in our example.

Taking advantage of the homogeneity of the unknown function $J$, it can be verified from equation (31) that $J$ can be written in the form: $S^{\gamma} H\left(\lambda^{1}, \lambda^{2}\right)$. Substituting this into the first order condition in (32) yields

$$
w=\frac{\eta-r_{t}}{\sigma^{2}(1-\gamma)}
$$

Since in equilibrium $w=1$, the above first order condition immediately implies that the equilibrium short rate is

$$
r=\eta-\sigma^{2}(1-\gamma)
$$

which is constant. Using this fact, and the existing results on Merton's portfolio problem with Kreps-Porteus utility (Svensson, 1989, Weil, 1990, and Schroder and Skiadas, 1997), the individual's utility is given by

$$
A_{n}\left(W_{t}^{n}\right)^{\gamma}
$$

where the constant $A_{n}$ is:

$$
A_{n}=\left[\left(1-\rho^{n}\right)\left(\beta^{n}-\rho^{n}\left(r-\frac{(\eta-r)^{2}}{\sigma^{2}(1-\gamma)}\right)\right)\right]^{1-1 / \rho^{n}}, \quad n=1, \ldots, N
$$

Also, at the efficient allocation,

$$
\lambda_{t}^{i} A_{i}\left(W_{t}^{i}\right)^{\gamma-1}=\lambda_{t}^{j} A_{j}\left(W_{t}^{j}\right)^{\gamma-1}
$$

Collecting the above results, and writing $W_{t}^{n}=\theta_{t}^{n} S_{t}$, where

$$
\begin{aligned}
\theta_{t}^{n} & =\frac{\left[\frac{A_{N} \lambda_{t}^{N}}{A_{n} \lambda_{t}^{n}}\right]^{1 /(\gamma-1)}}{1+\sum_{n=1}^{N-1}\left[\frac{A_{N} \lambda_{t}^{N}}{A_{n} \lambda_{t}^{n}}\right]^{1 /(\gamma-1)}}, \quad n=1, \ldots, N-1, \\
\theta_{t}^{N} & =1-\sum_{n=1}^{N-1} \theta_{t}^{n},
\end{aligned}
$$


we have the following closed-form expression for the value function:

$$
J\left(S_{t}, \lambda_{t}^{1}, \ldots, \lambda_{t}^{N}\right)=\left[\sum_{n=1}^{N} \lambda_{t}^{n} A_{n}\left(\theta_{t}^{n}\right)^{\gamma}\right] S_{t}^{\gamma}
$$

This closed-form solution can be used to analyze the effect, on the efficient allocation, of differences in the intertermporal elasticity of substitution across agents.

We should remark, and the careful reader may have already noticed, that the economy in the above example does not meet some of the assumptions underlying the theorems in this or the papers cited. For instance, the isoelastic felicity functions postulated in (29) may lead to an efficient allocation for which the time path of the discount rate is not within a closed set $[-a, a]$, as has been assumed above. ${ }^{22}$ Also, the derivative of the value function of the dynamic program, $J_{x}$, is not bounded, as assumed in Theorems 2.3 and 3.1. ${ }^{23}$ Finally, the endowment set defined by equation (30) (with $c_{s}^{n}=0$ ) is not bounded away from zero with probability one. ${ }^{24}$ Of course, these are only sufficient conditions for an equilibrium to exist. For the parametric case considered in the example, the existence of a solution can be shown using other methods: Schroder and Skiadas (1997) have shown that the Kreps-Porteus utility exists and Svensson (1989) and Schroder and Skiadas (1997) have also shown the existence of a solution to Merton's portfolio problem with Kreps-Porteus utility. Based on these results, the above derivation not only gives the closed-form solution to the social welfare function, but also shows, as a by-product, that the equilibrium exists.

\section{Conclusion}

Under certainty, and for the case of an economy with only one agent, Geoffard (1996) has extended time-additive utility by modeling the discount factor as a particular function of the state. He calls this more general class "variational utility." He also shows the equivalence between a special case of variational utility and recursive utility. In this paper, we have extended variational utility to a

\footnotetext{
${ }^{22}$ Stating the same issue in terms of the aggregator function $f$ of footnote 18 , it is clear that $f$ does not satisfy the growth and Lipschitz conditions. Duffie and Lions (1992) discuss how to address this problem in the Markovian setting.

${ }^{23}$ This is because the felicity function (29) itself does not have a bounded derivative with respect to consumption. Clearly that derivative approaches plus infinity as consumption approaches zero. In other words, the Inada condition holds.

${ }^{24}$ That is, the variable $S$ has a non zero probability of falling below any point no matter how close to zero that point has been chosen to be. While we have not assumed boundedness away from zero explicitly in any of our theorems, we have assumed the existence of a solution to problem (22)-(24) and Duffie, Geoffard and Skiadas (1994) have shown the existence of a solution only under this assumption.
} 
stochastic environment, and have shown that stochastic variational utility is equivalent to recursive utility under uncertainty.

Our main contribution is to have shown how to use variational utility to characterize Paretoefficient allocations in a multiagent economy where the individual agents have recursive utility. This characterization of efficient allocations is in terms of a single value function rather than a value function for each investor in the economy. Moreover, we have shown that one can solve for the single value function using the familiar technique of stochastic dynamic programming. 


\section{A The Uncertainty Case in Discrete Time}

In this appendix, we show heuristically that the discrete-time efficiency problem expressed in the form of variational utility is equivalent to the efficiency problem expressed in the form of a recursive utility with a certainty equivalent operator equal to the expected value.

We start with the discrete-time analog of the welfare optimizing problem under uncertainty, equation (25):

$$
J(x, \lambda) \equiv \max _{\left\{c_{t}^{n}\right\}} \min _{\left\{\nu_{t}^{n}\right\}} E_{t} \sum_{s \geq t} \sum_{n=1}^{N}\left[\lambda^{n}(s) F^{n}\left(c_{s}^{n}, \nu_{s}^{n}\right)\right],
$$

subject to feasibility constraints, and:

$$
\lambda_{t+1}^{n}=\left[1-\nu_{t}^{n}\right] \lambda_{t}^{n} ; n=1, \ldots, N
$$

The Bellman equation for this problem is: ${ }^{25}$

$$
J(x, \lambda)=\max _{\left\{c^{n}\right\}} \min _{\left\{\nu^{n}\right\}}\left\{\sum_{n=1}^{N} \lambda^{n} F^{n}\left(c^{n}, \nu^{n}\right)+E_{t} J\left[x_{t+1}, \lambda(1-\nu)\right]\right\},
$$

where we have used the shorthand: $\lambda(1-\nu) \equiv\left(\lambda^{1}\left(1-\nu^{1}\right), \ldots, \lambda^{N}\left(1-\nu^{N}\right)\right)$. The associated first-order conditions for the min problem are:

$$
0=F_{\nu^{n}}^{n}\left(c^{n}, \nu^{n}\right)-E_{t} J_{\lambda^{n}}\left[x_{t+1}, \lambda(1-\nu)\right]
$$

Differentiate (A1) with respect to each $\lambda^{n}$ to get:

$$
J_{\lambda^{n}}(x, \lambda)=F^{n}\left(c^{n}, \nu^{n}\right)+\left(1-\nu^{n}\right) E_{t} J_{\lambda^{n}}\left[x_{t+1}, \lambda(1-\nu)\right]
$$

In these equations, we impose that (A2) be satisfied by a proper choice of $\left\{\nu^{n}\right\} .{ }^{26}$ Now, define the functions $V^{n}$ :

$$
V^{n}(x, \lambda) \equiv J_{\lambda^{n}}(x, \lambda),
$$

\footnotetext{
${ }^{25}$ By virtue of the obvious homogeneity of degree 1 of the function $J(x, \lambda)$ with respect to $\left\{\lambda^{n}\right\}$, we could rewrite (A1) as:

$$
J(x, \lambda)=\max _{\left\{c^{n}\right\}} \min _{\left\{\nu^{n}\right\}} \sum_{n=1}^{N} \lambda^{n}\left\{F^{n}\left(c^{n}, \nu^{n}\right)+\left(1-\nu^{n}\right) E_{t} J_{\lambda^{n}} x_{t+1}\right\} .
$$
}

That would make it look similar to (28).

${ }^{26}$ This does not mean that:

$$
J_{\lambda^{n}}(x, \lambda)=\min _{\left\{\nu^{n}\right\}}\left\{F^{n}\left(c^{n}, \nu^{n}\right)+\left(1-\nu^{n}\right) E_{t} J_{\lambda^{n}}\left[x_{t+1}, \lambda(1-\nu)\right]\right\} .
$$


and the functions $W^{n}$ :

$$
W^{n}(c, u) \equiv \min _{\nu}\left[F^{n}(c, \nu)+(1-\nu) u\right]
$$

Then, the system of equations (A3) with the associated conditions (A2) imply that:

$$
V^{n}(x, \lambda)=W^{n}\left\{c^{n}, E_{t} V^{n}\left[x_{t+1}, \lambda(1-\nu)\right]\right\}
$$

an equation which shows that there exists an aggregator function $W^{n}$ and a value function $V^{n}$ for each individual $n$, which define for this agent a recursive utility functional in discrete time. Each step of the above heuristic proof is reversible so that equivalence is shown.

Remark: Equation (A4) defines a special form of recursive utility, viz. the form of recursive utility in which the certainty equivalent operator is the expected value. In continuous time (Section 3.2), this was without loss of generality since Duffie and Epstein (1992) have shown how aggregators can be normalized so that the corresponding certainty equivalent is the expected value. In discrete time, we are not aware of a similar result.

Remark: If the aggregator function $W(c, u)$ is of the CES type:

$$
W(c, u)=\left[c^{\rho}+\beta(\gamma u)^{\frac{\rho}{\gamma}}\right]^{\frac{\gamma}{\rho}} \frac{1}{\gamma}
$$

then the corresponding felicity function, $F(c, \nu) \equiv \max _{u}[W(c, u)-(1-\nu) u]$, is:

$$
F(c, \nu)=\frac{c^{\gamma}}{\gamma}\left\{\left[1+\beta\left[\left(\frac{1-\nu}{\beta}\right)^{\frac{\gamma}{\gamma-\rho}}-\beta\right]^{-1}\right]^{\frac{\gamma}{\rho}}-(1-\nu)\left[\left(\frac{1-\nu}{\beta}\right)^{\frac{\rho}{\gamma-\rho}}-\beta\right]^{-\frac{\gamma}{\rho}}\right\}
$$




\section{References}

Constantinides, G. (1982): "Intertemporal Asset Pricing with Heterogeneous Consumers and without Demand Aggregation," Journal of Business, 55, 253-267.

Dana, R. and C. Le Van (1990), "Structure of Pareto Optima in an Infinite-Horizon Economy where Agents Have Recursive Preferences," Journal of Optimization Theory and Applications, 64, 269-292.

Duffie, D. (1996): Dynamic Asset Pricing Theory, Princeton University Press.

Duffie, D. and L. G. Epstein (1992): "Stochastic Differential Utility," Econometrica, 60, 353-394.

Duffie, D., P.-Y. Geoffard and C. Skiadas (1994): "Efficient and Equilibrium Allocations with Stochastic Differential Utility," Journal of Mathematical Economics, 23, 133-146.

Duffie, D. and P.-L. Lions, (1992), "PDE Solutions of Stochastic Differential Utility," Journal of Mathematical Economics, 21, 577-606.

Epstein, L. G. (1987): “The Global Stability of Efficient Intertemporal Allocations," Econometrica, 55, 329-355.

Epstein, L. G., and S. Zin (1989): "Substitution, Risk Aversion and the Temporal Behavior of Consumption and Asset Returns: A Theoretical Framework," Econometrica, 57, 937-969.

Fleming, W. and R. Rishel (1975): Deterministic and Stochastic Optimal Control, Berlin: Springer-Verlag.

Geoffard, P.-Y. (1996): "Discounting and Optimizing: Capital Accumulation as a Variational Minmax Problem," Journal of Economic Theory, 69, 53-70.

Kan, R. (1995): "Structure of Pareto Optima When Agents Have Stochastic Recursive Preferences," Journal of Economic Theory, 66, 626-631.

Koopmans, T. C. (196): "Stationary Ordinal Utility and Impatience," Econometrica, 28, 287-309.

Lucas, R. and N. Stokey (1984): "Optimal Growth with Many Consumers," Journal of Economic Theory, 32, 139-171.

Lucas, R. and N. Stokey (1989): Recursive Methods in Economic Dynamics, Harvard University Press.

Ma, J., P. Protter and J. Yong (1994): "Solving Forward-Backward Stochastic Differential Equations Explicitly-A Four Step Scheme," Probability Theory and Related Fields, 98, 339-359.

Magill, M. (1981): "An Equilibrium Existence Theorem," Journal of Mathematical Analysis and Applications, 84, 162-169.

Mas-Colell, A. (1986): "The Price Equilibrium Existence Problem in Topological Vector Lattices," Econometrica, 54, 1039-1054.

Negishi, T. (1960): "Welfare Economics and Existence of an Equilibrium for a Competitive Economy," Metroeconomica, 12, 92-97.

Growth," 
Protter, P. (1990): Stochastic Integration and Differential Equations, New York: Springer Verlag.

Rockafellar, R. T. (1970): Convex Analysis, Princeton University Press.

Schroder, M. and C. Skiadas (1997): "Optimal Consumption and Portfolio Selection with Stochastic Differential Utility," Northwestern University working paper.

Svensson, L. (1989): "Portfolio Choice with Non-Expected Utility in Continuous Time," Economic Letters, 30, 313-317.

Weil, P. (1990): "Nonexpected Utility in Macroeconomics," Quarterly Journal of Economics, 105, $29-42$. 